\title{
Hérnia perineal em ovino com útero gravídico como conteúdo
}

\section{Perineal hernia in sheep containing pregnancy uterus}

\author{
Roberta Garbelini Gomes ${ }^{1}$; Mariana Cosenza ${ }^{1}$; \\ Júlio Augusto Naylor Lisbôa²; Augusto José Savioli de Almeida Sampaio²*
}

\section{Resumo}

Herniações e eventrações são ocorrências comuns na clinica buiátrica. Hérnia umbilical e inguinoescrotal são bem descritas, porém hérnias perineais em pequenos ruminantes são pouco relatadas. Apesar de a hérnia perineal ser mais comum em cães e gatos, pode ser ocasionalmente observada em ovelhas gestantes próximas ao parto sendo que raramente apresenta o útero como conteúdo. O presente relato descreve a ocorrência de hérnia perineal com útero gravídico como conteúdo em uma ovelha de aproximadamente um ano de idade sem raça definida com tempo de gestação aproximado de 120 dias. A terapêutica adotada incluiu a redução cirúrgica da hérnia, porém, sem a síntese do anel herniário. $\mathrm{O}$ animal apresentou excelente recuperação sem complicações pós-operatórias.

Palavras-chave: Hérnia perineal, paresia, ovino

\begin{abstract}
Herniations and eventrations are ordinary events of buiatric clinic. Umbilical and inguinal hernias are well described, but perineal hernias in small ruminant animals are not so mentioned. Even though being the perineal hernia more common in cats and dogs, it might be occasionally observed in pregnant sheep close to delivery, being the gravid uterus rarely as content. The present study describes the event of perineal hernia with gravid uterus as content in a sheep of approximately one year old with no breed defined with a pregnancy period about 120 days. The adopted procedure included surgical reduction of the hernia, but without the closure of the hernial ring. The animal presented excellent recovery without post-surgery complications.
\end{abstract}

Key words: Perineal hernia, paresis, sheep

\footnotetext{
${ }^{1}$ Residentes da Clínica Médica, Cirúrgica e Reprodução de Grandes Animais - Universidade Estadual de Londrina. E-mail: robs_ vet@hotmail.com; maricosenza@yahoo.com.br

${ }^{2}$ Docentes do Departamento de Clínicas Veterinárias - Universidade Estadual de Londrina. DCV - CCA - C.P. 6001 - 86051-990, Londrina, PR. E-mail: asampaio@uel.br; janlisboa@uel.br

* Autor para correspondência
} 


\section{Introdução}

O termo hérnia se refere aos órgãos que se deslocam de sua localização anatômica normal para uma cavidade neoformada ou natural através de um orifício anatomicamente fraco. As hérnias comumente encontradas nos animais domésticos são: hérnia diafragmática, hérnia inguinal, hérnia escrotal, hérnia umbilical, hérnia abdominal traumática, hérnia hiatal, hérnia incisional e hérnia perineal (HUNT, 2006).

As hérnias podem ser classificadas de acordo com a freqüência, época de aparecimento, estrutura, percurso, relação anatômica, conteúdo, localização e topografia (SOUZA; ABILIO, 2007). De acordo com as estruturas, as hérnias podem ser classificadas em falsas ou verdadeiras. As hérnias falsas possuem o saco formado pela pele, subcutâneo, fáscia ou qualquer outra estrutura; já nas verdadeiras, o saco é constituído de peritôneo parietal. Nas hérnias perineais, o saco herniário não costuma ser constituído de peritôneo, o que faz com que sejam classificadas como hérnias falsas. Essa afecção é mais conhecida como ruptura do diafragma pélvico (SOUZA; ABILIO, 2007).

O diafragma pélvico é o fechamento vertical do canal pélvico através do qual passa o reto, sendo composto pelos músculos coccígeo e elevador anal e pelas fáscias interna e externa (MANN; CONTANTINESCU, 2005; MORTARI; RAHAL, 2005).

A hérnia perineal é o resultado de um enfraquecimento e separação dos músculos e fáscias que envolvem o diafragma pélvico. Em geral, a hérnia perineal ocorre entre os músculos esfíncter externo do ânus e elevador do ânus, ocasionalmente entre os músculos elevador do ânus e coccígeo. A exata causa da fraqueza muscular é desconhecida, mas vários fatores já foram propostos. A fraqueza muscular poderá ocasionar desvios de órgãos intraabdominais caudalmente e desvio ou dilatação do reto dentro do períneo. Os fatores que exacerbam a presença da hérnia perineal em animais de companhia são enterites, prostatomegalia e megacolon (LÓPEZ et al., 2004; MANN; CONTANTINESCU, 2005; MORTARI; RAHAL, 2005; HUNT, 2006).

A ocorrência de hérnia perineal em ovinos, tendo como conteúdo o útero não é comum, porém, essa condição já foi descrita em caprinos, humanos, gatos, cães, vacas, búfalos e macacos (ROBERTS, 1986a; MOBINI; HEATH; PUGH, 2004; SOUZA; ABILIO, 2007). Em ovinos pode ser ocasionalmente observada em ovelhas gestantes próximas ao parto sendo que raramente apresenta o útero como conteúdo. Caso isso ocorra, esta afecção pode interferir na eficiência do parto e às vezes julga-se necessária a assistência devido à ocorrência de distocias, nas quais poderão ocorrer lesões nos nervos ciático, obturador e fibular. Essas lesões nervosas são muito mais comuns em bovinos e raramente ocorrem em ovinos e caprinos (ROBERTS, 1986b; JACKSON, 1997; MAXIE-SMITH, 1997; CONSTABLE, 2004; DIVERS, 2004; MANN; CONTANTINESCU, 2005). Além disso, essa condição tem sido freqüentemente observada em decorrência de injeções intramusculares, miopatia isquêmica, compressão por neoplasia, abscessos ou fraturas de pelve (DIVERS, 2004).

O diagnóstico de hérnia perineal baseia-se na anamnese, exame físico (sinais clínicos e palpação externa ou retal). Radiografia e ultra-sonografia são métodos de auxílio ao diagnóstico (GRUNERT; BIRGEL, 1982; MANN; CONTANTINESCU, 2005; MORTARI; RAHAL, 2005; SOUZA; ABILIO, 2007).

Como diagnósticos diferencias podem ser citados hematoma, abscesso, flegmão, neoplasia anal, perineal e retal, atresia anal e tumores vaginais (FOSSUM, 2002).

Em grandes animais, o reparo cirúrgico das hérnias perineais não é normalmente realizado (JACKSON, 1997). 


\section{Relato de Caso}

Umaovelhasemraçadefinidadeaproximadamente um ano de idade, pesando $34 \mathrm{~kg}$, foi atendida no Hospital Veterinário da Universidade Estadual de Londrina (UEL) com histórico de decúbito esternal há três dias, depressão e aumento de volume na região perineal.

Ao exame clínico observou-se depressão, anorexia, hipomotilidade ruminal, paraparesia flácida, estado de nutrição ruim, prenhez positiva de aproximadamente 120 dias e aumento de volume da região perineal de consistência dura, não redutível e não doloroso.

Através da vaginoscopia, foi observado cérvix fechado, com a presença de tampão mucoso, com a forma C (cônica), abertura 0 (fechada) e coloração C (hiperêmica) de acordo com a classificação de PRESTES (2004).

O hemograma do animal apresentou 28,8\% de hematócrito, $10,8 \mathrm{~g} / \mathrm{dL}$ de hemoglobina, $11,5 \times 10^{6}$ $/ \mathrm{mm}^{3}$ de hemáceas, 8.440 leucócitos $/ \mathrm{mm}^{3}$ sendo que $84 \%$ de segmentados e $16 \%$ linfócitos, 7,6 $\mathrm{g} / \mathrm{dL}$ proteína total plasmática e $600 \mathrm{mg} / \mathrm{dL}$ de fibrinogênio.

No exame radiográfico da região abdominal e perineal foi detectada gestação gemelar, sendo que a cabeça de um dos fetos projetava-se pela região pélvica e insinuava-se pelo períneo, formando um saco herniário. Diante do observado foi estabelecido o diagnóstico de hérnia perineal com útero gravídico como conteúdo.

Em virtude da suspeita de toxemia da prenhez apresentada por esse animal optou-se por realizar fluidoterapia com 4 litros de solução de ringer com lactato acrescentando-se a cada frasco de $500 \mathrm{ml}, 50$ $\mathrm{ml}$ de glicose a $50 \%$ e $5 \mathrm{ml}$ de gluconato de cálcio a $20 \%$.

Devido à impossibilidade de redução externa da hérnia perineal e a toxemia gravídica confirmada pela resposta transitória ao tratamento, optou-se por realizar a laparotomia pelo flanco esquerdo seguida de cesariana, sendo que nenhum dos dois fetos se apresentava viável.

No pós-operatório foram administradas 30000UI/ $\mathrm{kg}$ de penicilina benzatina por via intramuscular, sendo realizada uma aplicação a cada 48 horas, totalizando três aplicações, dexametasona (1mg/ $\mathrm{kg}$ ) por via intramuscular em dose única, cloridrato de tiamina $(20 \mathrm{mg} / \mathrm{kg})$ por via intramuscular a cada 24 horas, totalizando quatro aplicações, cipionato de estradiol (1mg) por via intramuscular em dose única e flunixina meglumina $(1,1 \mathrm{mg} / \mathrm{kg})$ por via intramuscular em dose única.

Durante três dias o animal foi mantido em estação por períodos de 12 horas com o auxílio de aparato suspensor devido à paraparesia flácida apresentada. No $10^{\circ}$ dia de pós-operatório, o animal mantinha-se em estação conseguindo suportar o peso e caminhar, com apetite e alerta, obtendo alta.

\section{Resultados e Discussão}

A ocorrência de hérnia perineal em pequenos ruminantes não é comum, porém essa afecção foi reportada por Saperstein (1993) em dois cordeiros machos da raça merino e em um bezerro, estando associada com algumas malformações, incluindo atresia anal (cordeiro e bezerro) e hérnia diafragmática (cordeiro).

A etiologia desta afecção ainda é desconhecida apesar de alguns estudos destacarem causas predisponentes e ocasionais na ocorrência de hérnia durante a gestação como fatores hereditários e disposição anatômica; distensão exagerada dos cornos uterinos, como pode ocorrer na hidropsia dos envoltórios fetais e na gravidez múltipla patológica; causas mecânicas ou traumáticas como após salto, quedas ou transportes (GRUNERT; BIRGEL, 1982; SAPERSTEIN, 1993).

O histórico clínico do animal caracterizado por aumento de volume perineal, não redutível, não doloroso, assim como o exame físico do mesmo, se mostram em consonância com as descrições prévias 
da enfermidade (MANN; CONTANTINESCU, 2005; MORTARI; RAHAL, 2005; SOUZA; ABILIO, 2007). No entanto, as hérnias perineais são mais comumente observadas em pequenos animais, principalmente nos machos entre sete e nove anos de idade, caracterizando-se pela ruptura do diafragma pélvico, com subseqüente herniação do reto e órgãos pélvicos dentro da fossa ísquio-retal (SILVA; REZENDE; OLIVEIRA, 1996; LÓPEZ et al., 2004; MANN; CONTANTINESCU, 2005; MORTARI; RAHAL, 2005). López et al. (2004) relata que fêmeas caninas são menos susceptíveis à ocorrência de hérnia perineal pelo fato de possuírem o diafragma pélvico mais forte para suportar a força do parto, porém, tal relação em se tratando da espécie ovina, ainda precisa ser estabelecida.

A retroflexão da bexiga é um achado comum em pequenos animais com esta afeç̧ão, o que freqüentemente leva ao desenvolvimento de estrangúria, disúria e anúria (LÓPEZ et al., 2004; MANN; CONTANTINESCU, 2005; MORTARI; RAHAL, 2005). No entanto, no caso relatado não foram observadas estas complicações.

Lesões nos nervos ciáticos em ruminantes podem ocorrer por traumas durante o parto, injeções intramusculares, miopatia isquêmica, compressão por neoplasia, abscesso e fratura de pelve (MAXIESMITH, 1997; REBHUN; GUARD; RICHARDS, 2000; DIVERS, 2004; MOBINI; HEATH; PUGH, 2004). As distocias (especialmente nas primíparas ou fêmeas com fetos exageradamente grandes) podem levar à compressão dos nervos obturador e ciático. Devido à anatomia, esses nervos encontram-se vulneráveis a lesões compressivas durante a distocia (MAXIE-SMITH, 1997; REBHUN; GUARD; RICHARDS, 2000; DIVERS, 2004). No entanto, devido ao porte dos pequenos ruminantes, as lesões nos nervos periféricos são menos comuns, sendo que algumas podem acontecer nos casos de mordidas de predadores ou seguida de administração de drogas intramusculares (GRUNERT; BIRGEL, 1982; TONIOLLO; VICENTE, 2003; CONSTABLE, 2004; DIVERS, 2004). Diferentemente das causas descritas acima, acredita-se que o conteúdo herniário formado pelo feto insinuado, comprimiu os nervos ciático e obturador, provocando a paraparesia flácida, a qual foi debelada com medicação antiinflamatória, aparato suspensor e fisioterapia.

Além das lesões nos nervos ciático e obturador, a incontinência fecal, prolapso retal, incontinência urinária e necrose da bexiga podem ser outras complicações em animais que apresentarem essa enfermidade (MANN; CONTANTINESCU, 2005), porém, esses problemas não foram observados nesse animal.

Em virtude da toxemia da gestação também apresentada pelo animal e o conteúdo da hérnia ser o útero gravídico, a laparotomia seguida de cesariana foi o método de escolha (GRUNERT; BIRGEL, 1982; TONIOLLO; VICENTE, 2003). A toxemia da gestação em pequenos ruminantes ocorre no terço final da gestação, em fêmeas com fetos múltiplos que foram submetidas a péssimas condições de alimentação, o que é confirmado pelas condições apresentadas pelo animal. A hipocalcemia é uma afecção que pode ocorrer concomitantemente. Todos esses sinais corroboram os que foram apresentados pelo animal, o qual obteve melhora transitória logo após a administração de fármacos. A remoção do feto o mais rápido possível deve ser realizada e nos casos críticos a cesariana é indicada (MOBINI; HEATH; PUGH, 2004; PEARSON apud SMITH, 2006).

O tratamento conservador das hérnias perineais em cães não parece apresentar bons resultados a longo prazo, seja pelo fato de apresentarem agravamento dos sintomas, seja pelo perigo de ocorrer retroflexão da bexiga a qualquer momento. A recidiva da hérnia perineal em cães pode ocorrer em 10 a $46 \%$ dos casos e para diminuir essa incidência algumas técnicas cirúrgicas estão sendo empregadas na reconstituição do diafragma pélvico. A transposição do músculo obturador interno, a transposição do músculo glúteo superficial e a transposição do músculo semitendinoso são técnicas 
cirúrgicas muito empregadas (SILVA; REZENDE; OLIVEIRA, 1996; LÓPEZ et al., 2004; MANN; CONTANTINESCU, 2005; MORTARI; RAHAL, 2005; SOUZA; ABILIO, 2007). No entanto, ao contrário do relatado na literatura em relação a pequenos animais, a terapêutica adotada sem a síntese do anel herniário apresentou excelente resultado, o que corrobora o afirmado por Jackson (1997).

Dentre os fatores a se considerar quanto ao prognóstico da hérnia perineal, podem ser destacados o tamanho da hérnia, o tempo de formação da hérnia até sua correção cirúrgica e as características do conteúdo (SOUZA; ABILIO, 2007). Sem a síntese do anel herniário e com o tratamento complementar o caso apresentou boa evolução e com prognóstico favorável. Sendo o paciente submetido à alta em poucos dias.

\section{Conclusão}

O presente relato descreve um caso de hérnia perineal em pequenos ruminantes (ovino) apresentando o útero gravídico como conteúdo, na qual a terapêutica empregada, sem a necessidade de síntese do anel herniário, possibilitou resultados satisfatórios. No entanto, cabe a ressalva que maiores observações devem ser feitas quanto a possibilidade de recidiva do processo a longo prazo.

\section{Referências}

CONSTABLE, P. D. Clinical examination of the ruminant nervous system. The Veterinary Clinics of North America: Food Animal Practice, U.S.A., v. 20, n. 2, p. 185-214, 2004.

DIVERS, T. J. Acquired spinal cord and peripheral nerve disease. In: CONSTABLE, P. D. The Veterinary Clinics of North America: Food Animal Practice, U.S.A., v. 20, n. 2, p. 231-242, 2004.

FOSSUM, T. W. Surgery of the digestive system. In:_. Small animal surgery. U.S.A.: Ed. Mosby, 2002. p. 274-449.
GRUNERT, E.; BIRGEL, E. H. Obstetrícia veterinária. Porto Alegre: Ed. Sulina, 1982.

HUNT, G. B. Hernia repair: principles \& practices. North American Veterinary Conference, Sidney, Austrália. 2006.

JACKSON, P. G. G. Problemas na gestação. In: Obstetrícia veterinária. Brasil: Ed. Roca, 1997.

LÓPEZ, J. E.; GUAIMÁS MOYA, L. E.; BÁEZ, A. D.; LOCKETT, M. B.; LUDUENO, S. F.; RESOAGLI, J. M.; HOLOVATE, R.; AMARILLA, O. A.; MAIDANA, H. R.; LÓPEZ RAMOS, M. L. Tratamiento quirúrgico de las hérnias perineales mediante el uso injerto de pericardio conservado en glicerina al 98\% (resultados preliminares). Comunicaciones Científicas y Tecnológicas Universidad Nacional del Nordeste, Argentina, 2004.

MANN, F. A.; CONTANTINESCU, G. M. Salvage techniques for failed perineal herniorrhaphy. In: BOJRAB, M. J.; ELLISON, G. W.; SLOCUM, B. Current techniques in small animal surgery. [S.1.: s.n.], 2005. p. 564-570.

MAXIE-SMITH, L. Diseases of nervous system. In: GREENOUGH, P. R. Lameness in cattle. Philadelphia: Ed. W. B. Saunders Company, 1997. p. 206-213.

MOBINI, S.; HEATH, A. M.; PUGH, D. G. Teriogenologia de ovinos e caprinos. In: PUGH, D. G. Clínica de ovinos e caprinos. São Paulo. Ed. Roca, 2004. p. 145-208.

MORTARI, A. C.; RAHAL, S. C. Hérnia perineal em cães. Revista Ciência Rural. Santa Maria, v. 35, n. 5, p. 1220-1228, 2005.

PEARSON, E. G. Enfermidades do sistema hepatobiliar. In: SMITH, B. P. Medicina interna de grandes animais. Barueri: Ed. Manole, 2006. p. 810-816.

PRESTES, N. C. Semiologia do sistema reprodutor feminino. In: FEITOSA, F. L. F. Semiologia veterinária. São Paulo: Ed. Roca, 2004, p. 335-348.

REBHUN, W. C.; GUARD, C.; RICHARDS, C. M. Doenças do gado leiteiro. São Paulo: Ed. Roca, 2000, p. 527-536.

ROBERTS, S. J. Diagnosis and treatment of various types of distocia. In: Veterinary obstetrics and genital diseases (theriogenology). 3.ed. Michigan: Ed. Edwards Brothers,1986b. p. 343-346.

Diseases and accidents of the gestation period. In:__ Veterinary obstetrics and genital diseases (theriogenology). 3.ed. Michigan: Ed. Edwards Brothers, 1986a. p. 228. 
SAPERSTEIN, G. Congenital abnormalities of internal organs and body cavities. In: STANLEY, M. D. The Veterinary Clinics of North America: Food Animal Practice, v. 9, n. 1, p. 115-125, 1993.

SILVA, M. C.; REZENDE, C. M. F.; OLIVEIRA, H. P. Herniorrafia perineal: transposição do músculo obturador interno. Arquivo Brasileiro de Medicina Veterinária e Zootecnia, Belo Horizonte, v. 48, n. 2, p. 147-155, 1996.

SOUZA, D. B.; ABILIO, E. J. Hérnia perineal em cães - revisão de literatura. Revista Clinica Veterinária, n. 68, ano12, p. 78-86, maio/jun. 2007.

TONIOLLO, G. H.; VICENTE, W. R. R. Manual de obstetrícia veterinária. São Paulo. Ed. Varela, 2003. 